\title{
Confusions in orbivirus protein classification
}

\author{
Meik Dilcher ${ }^{*}$ and Manfred Weidmann
}

\begin{abstract}
An extensive comparative analysis of orbivirus genomes revealed four cases of unclear numeration and protein designation, due to confused reference to protein size or segment size by which they are encoded. A concise nomenclature based on type species, sequence homology and functional characteristics independent of segment or protein size is suggested.
\end{abstract}

Keywords: Reoviridae, Orbivirus, Protein classification

\section{Background}

The genus Orbivirus is one of 15 in the family of Reoviridae containing 22 serogroups (species) and at least 160 different serotypes (strains) [1]. Orbiviruses are transmitted by insects (midges, flies, mosquitoes) or by ticks. Their double-stranded RNA (dsRNA) genomes consist of 10 segments coding for seven structural and at least three non-structural proteins. Orbiviruses have no envelope but a double-shelled icosahedral capsid [2] and include pathogenic agents of wild animals (Epizootic hemorrhagic disease virus (EHDV)), domestic animals (Bluetongue virus (BTV) and African horse sickness virus (AHSV)), and of man (Kemerovo virus (KEMV)) [3]. Type species of the genus is the Culicoides midge transmitted BTV. Insect-borne orbiviruses are much better characterized than tick-transmitted orbiviruses for which few sequences have been described: Broadhaven virus (BRDV, partial) [4], Sandy Bay virus ( $\mathrm{SBaV}$, partial (formerly Nugget virus)) [5-8], St Croix River virus (SCRV, complete genome) [9], Great Island virus (GIV, complete genome) [7].

Recently we determined the complete genomes of Tribeč virus (TRBV) and KEMV in a pyrosequencing approach [10] complementing available partial information on segments 1, 2 and 6 of these viruses and of Lipovnik virus (LIPV) [7].

During our extensive comparative analysis of orbivirus genomes we noticed four cases of unclear numeration and protein designation (see Tables 1 and 2). Some laboratories classify orbivirus proteins according to the

\footnotetext{
* Correspondence: meik.dilcher@medizin.uni-goettingen.de

Department of Virology, University Medical Center Göttingen, Kreuzbergring 57, D-37075 Göttingen, Germany
}

(C) 2012 Dilcher and Weidmann; licensee BioMed Central Ltd. This is an Open Access article distributed under the terms of the Creative Commons Attribution License (http://creativecommons.org/licenses/by/2.0), which permits unrestricted use distribution, and reproduction in any medium, provided the original work is properly cited. size of the proteins whereas others use the size of genome segments from which they are encoded.

(i) The inner shell protein T2 for example can be encoded by segment 2 (tick- and mosquito-borne orbiviruses) or segment 3 (Culicoides-borne orbiviruses). This leads to some laboratories labeling this protein VP2(T2) (e.g. GIV), while others designate it VP3(T2) as in the type species BTV. For Peruvian horse sickness virus (PHSV) however, the segment 2 encoded protein is designated VP3 (T2) although it is larger (925 amino acids) than the segment 3 encoded VP2 protein (881 amino acids) [11]. To avoid confusion with the outer shell protein VP2 we suggest to exclusively use VP3(T2) for all $\mathrm{T} 2$ proteins.

(ii) VP2 and VP2 homologous proteins can be encoded by segments 2, 3, 4 and 5 and are designated VP2, VP3 (YUOV, SCRV) or VP4 (BRDV segment 4 ([12,13], sequence entry to GenBank missing)), GIV segment 5). Because of the location on the outer capsid and the described sequence similarity with other VP2 proteins, we suggest that the VP4 proteins (BRDV, GIV) as well as the VP3 proteins (YUOV, SCRV) should be uniformly termed VP2, even though tick-borne VP2 proteins have only half the size of insect-borne VP2 proteins [13].

(iii) The capping enzyme VP4(CaP) can be encoded by segment 4 (BTV, YUOV, SCRV etc.) or segment 3 (TRBV, KEMV). In GIV this protein is designated VP3(CaP) [7] and should be renamed VP4(CaP) to avoid confusions with VP3(T2).

(iv) In most cases VP5 is encoded by segment 6 and comprises a component of the outer shell that 
Table 1 Comparison of the genome segments and encoded proteins of BTV, YUOV, TRBV, KEMV and GIV

\begin{tabular}{|c|c|c|c|c|c|c|c|c|c|c|c|}
\hline \multicolumn{4}{|c|}{ BTV (insect-transmitted) } & \multicolumn{4}{|c|}{ YUOV (insect-transmitted) } & \multicolumn{4}{|c|}{ TRBV (tick-transmitted) } \\
\hline Segment 1 & VP1 (Pol) & 150 kDa & $\begin{array}{l}\text { RNA-dep--RNA- } \\
\text { Polymerase }\end{array}$ & Segment 1 & VP1 (Pol) & 151 kDa & $\begin{array}{l}\text { RNA-dep.-RNA- } \\
\text { Polymerase }\end{array}$ & Segment 1 & VP1 (Pol) & $146 \mathrm{kDa}$ & $\begin{array}{l}\text { RNA-dep-RNA- } \\
\text { Polymerase }\end{array}$ \\
\hline 3944 bp & ACR58458 & $1302 \mathrm{AA}$ & & $3393 \mathrm{bp}$ & YP_443925 & $1315 \mathrm{AA}$ & & 3892 bp & HQ266581 & $1284 \mathrm{AA}$ & \\
\hline Segment 2 & VP2 & $111 \mathrm{kDa}$ & Outer shell & Segment 2 & VP2 (T2) & 107 kDa & Inner shell & Segment 2 & VP3 (T2) & $102 \mathrm{kDa}$ & Inner shell \\
\hline 2953 bp & ACR58459 & $956 \mathrm{AA}$ & & $2900 \mathrm{bp}$ & YP_443926 & $940 \mathrm{AA}$ & & $2793 \mathrm{bp}$ & HQ266582 & $908 \mathrm{AA}$ & \\
\hline Segment 3 & VP3 (T2) & 103 kDa & Inner shell & Segment 3 & VP3 & 100 kDa & Outer shell & Segment 3 & VP4 (CaP) & $72 \mathrm{kDa}$ & Capping Enzyme \\
\hline $2772 \mathrm{bp}$ & ACR58460 & $901 \mathrm{AA}$ & & $2688 \mathrm{bp}$ & YP_443927 & $873 \mathrm{AA}$ & & $1935 \mathrm{bp}$ & HQ266583 & $628 \mathrm{AA}$ & \\
\hline Segment 4 & VP4 (CaP) & $75 \mathrm{kDa}$ & Capping Enzyme & Segment 4 & VP4 (CaP) & $74 \mathrm{kDa}$ & Capping Enzyme & Segment 4 & NS1 (TuP) & $62 \mathrm{kDa}$ & Formes Tubules \\
\hline $1980 \mathrm{bp}$ & ACR58461 & $644 \mathrm{AA}$ & & 1993 bp & YP_443928 & $645 \mathrm{AA}$ & & $1734 \mathrm{bp}$ & HQ266584 & $529 \mathrm{AA}$ & \\
\hline Segment 5 & NS1 (TuP) & $64 \mathrm{kDa}$ & Forms Tubules & Segment 5 & NS1 (TuP) & $67 \mathrm{kDa}$ & Forms Tubules & Segment 5 & VP2 & $62 \mathrm{kDa}$ & Outer shell \\
\hline 1769 bp & ACR58463 & $552 \mathrm{AA}$ & & 1957 bp & YP_443929 & $574 \mathrm{AA}$ & & $1730 \mathrm{bp}$ & HQ266585 & $554 \mathrm{AA}$ & \\
\hline Segment 6 & VP5 & $59 \mathrm{kDa}$ & Outer shell & Segment 6 & VP5 & $59 \mathrm{kDa}$ & Outer shell & Segment 6 & VP5 & $59 \mathrm{kDa}$ & Outer shell \\
\hline 1638 bp & ACR58462 & $526 \mathrm{AA}$ & & $1683 \mathrm{bp}$ & YP_443930 & $535 \mathrm{AA}$ & & $1668 \mathrm{bp}$ & HQ266586 & $537 \mathrm{AA}$ & \\
\hline Segment 7 & VP7 (T13) & $39 \mathrm{kDa}$ & Inner shell & Segment 7 & NS2 (ViP) & $48 \mathrm{kDa}$ & Viral inclusion body & Segment 7 & NS2 (ViP) & $41 \mathrm{kDa}$ & Viral inclusion body \\
\hline 1156 bp & ACR58464 & 349 AA & & 1504 bp & YP_443931 & $435 \mathrm{AA}$ & & 1196 bp & HQ266587 & $368 \mathrm{AA}$ & \\
\hline Segment 8 & NS2 (ViP) & $41 \mathrm{kDa}$ & Viral inclusion body & Segment 8 & VP7 (T13) & $40 \mathrm{kDa}$ & Inner shell & Segment 8 & VP7 (T13) & $40 \mathrm{kDa}$ & Inner shell \\
\hline $1125 \mathrm{bp}$ & ACR58465 & 354 AA & & $1191 \mathrm{bp}$ & YP_443932 & $355 \mathrm{AA}$ & & $1184 \mathrm{bp}$ & HQ266588 & $357 \mathrm{AA}$ & \\
\hline Segment 9 & VP6 (Hel) & $36 \mathrm{kDa}$ & ssRNA and dsRNA & Segment 9 & VP6 (Hel) & $37 \mathrm{kDa}$ & ssRNA and dsRNA & Segment 9 & VP6 (Hel) & $33 \mathrm{kDa}$ & ssRNA and dsRNA \\
\hline 1049 bp & ACR58466 & 329 AA & Dinain & 1082 bp & YP_443933 & $338 \mathrm{AA}$ & Dine & 1034 bp & HQ266589 & $312 \mathrm{AA}$ & \\
\hline Segment 10 & NS3 & $26 \mathrm{kDa}$ & Glycoprotein & Segment 10 & NS3 & $28 \mathrm{kDa}$ & Glycoprotein & Segment 10 & NS3 & $23 \mathrm{kDa}$ & Glycoprotein \\
\hline 822 bp & ACR58467 & 229 AA & & $825 \mathrm{bp}$ & YP_443934 & $253 \mathrm{AA}$ & & $705 \mathrm{bp}$ & HQ266590 & $214 \mathrm{AA}$ & \\
\hline
\end{tabular}




\begin{tabular}{|c|c|c|c|c|c|c|c|}
\hline \multicolumn{4}{|c|}{ KEMV (tick-transmitted) } & \multicolumn{4}{|c|}{ GIV (tick-transmitted) } \\
\hline Segment 1 & VP1 (Pol) & 146 kDa & $\begin{array}{l}\text { RNA-dep.-RNA- } \\
\text { Polymerase }\end{array}$ & Segment 1 & VP1 (Pol) & 147 kDa & \multirow[t]{2}{*}{$\begin{array}{l}\text { RNA-dep.-RNA- } \\
\text { Polymerase }\end{array}$} \\
\hline 3896 bp & HQ266591 & 1285 AA & & 3897 bp & ADM88592 & $1285 \mathrm{AA}$ & \\
\hline Segment 2 & VP3 (T2) & 103 kDa & Inner shell & Segment 2 & VP2 (T2) & 103 kDa & \multirow[t]{2}{*}{ Inner shell } \\
\hline 2792 bp & HQ266592 & $908 \mathrm{AA}$ & & $2794 \mathrm{bp}$ & ADM88593 & $908 \mathrm{AA}$ & \\
\hline Segment 3 & VP4 (CaP) & $72 \mathrm{kDa}$ & Capping Enzyme & Segment 3 & VP3 (CaP) & $73 \mathrm{kDa}$ & \multirow[t]{2}{*}{ Capping Enzyme } \\
\hline 1934 bp & HQ266593 & $632 \mathrm{AA}$ & & 1936 bp & ADM88594 & $635 \mathrm{AA}$ & \\
\hline Segment 4 & VP2 & $63 \mathrm{kDa}$ & Outer shell & Segment 4 & NS1 (TuP) & $60 \mathrm{kDa}$ & \multirow[t]{2}{*}{ Formes Tubules } \\
\hline $1730 \mathrm{bp}$ & HQ266594 & $554 \mathrm{AA}$ & & 1731 bp & ADM88595 & $531 \mathrm{AA}$ & \\
\hline Segment 5 & NS1 (TuP) & $60 \mathrm{kDa}$ & Formes Tubules & Segment 5 & VP4 & $62 \mathrm{kDa}$ & \multirow[t]{2}{*}{ Outer shell } \\
\hline 1719 bp & HQ266595 & $529 \mathrm{AA}$ & & 1722 bp & ADM88596 & $551 \mathrm{AA}$ & \\
\hline Segment 6 & VP5 & $59 \mathrm{kDa}$ & Outer shell & Segment 6 & VP5 & $60 \mathrm{kDa}$ & \multirow[t]{2}{*}{ Outer shell } \\
\hline 1668 bp & HQ266596 & $537 \mathrm{AA}$ & & 1666 bp & ADM88597 & $537 \mathrm{AA}$ & \\
\hline Segment 7 & NS2 (ViP) & $41 \mathrm{kDa}$ & \multirow{2}{*}{$\begin{array}{l}\text { Viral inclusion body } \\
\text { matrix protein }\end{array}$} & Segment 7 & VP7 (T13) & $40 \mathrm{kDa}$ & \multirow[t]{2}{*}{ Inner shell } \\
\hline 1197 bp & HQ266597 & $368 \mathrm{AA}$ & & 1181 bp & ADM88598 & $357 \mathrm{AA}$ & \\
\hline Segment 8 & VP7 (T13) & $40 \mathrm{kDa}$ & Inner shell & Segment 8 & NS2 (ViP) & $39 \mathrm{kDa}$ & \multirow{2}{*}{$\begin{array}{l}\text { Viral inclusion body } \\
\text { matrix protein }\end{array}$} \\
\hline $1183 \mathrm{bp}$ & HQ266598 & $357 \mathrm{AA}$ & & 1172 bp & ADM88599 & $359 \mathrm{AA}$ & \\
\hline Segment 9 & VP6 (Hel) & $34 \mathrm{kDa}$ & \multirow{2}{*}{$\begin{array}{l}\text { ssRNA and dsRNA } \\
\text { binding helicase }\end{array}$} & Segment 9 & VP6 (Hel) & $34 \mathrm{kDa}$ & \multirow{2}{*}{$\begin{array}{l}\text { ssRNA and dsRNA } \\
\text { binding helicase }\end{array}$} \\
\hline 1049 bp & HQ266599 & $317 \mathrm{AA}$ & & 1056 bp & AMD88600 & $321 \mathrm{AA}$ & \\
\hline Segment 10 & NS3 & $23 \mathrm{kDA}$ & Glycoprotein & Segment 10 & NS3 & $19 \mathrm{kDa}$ & \multirow[t]{2}{*}{ Glycoprotein } \\
\hline 707 bp & HQ266600 & $214 \mathrm{AA}$ & & 703 bp & ADM88602 & $171 \mathrm{AA}$ & \\
\hline
\end{tabular}

Outer and inner shell proteins are labeled in bold. GenBank and SwissProt accession numbers are indicated. 
Table 2 Comparison of the genome segments and encoded proteins of SCRV, PHSV, BRDV and LIPV

\begin{tabular}{|c|c|c|c|c|c|c|c|}
\hline \multicolumn{4}{|c|}{ SCRV (tick-transmitted) } & \multicolumn{4}{|c|}{ PHSV (isolates only known from horses) } \\
\hline Segment 1 & VP1 (Pol) & $151 \mathrm{kDa}$ & RNA-dep.-RNA-Polymerase & Segment 1 & VP1 (Pol) & $151 \mathrm{kDa}$ & RNA-dep.-RNA-Polymerase \\
\hline 4089 bp & YP_052942 & 1345 AA & & 3987 bp & YP_460038 & $1311 \mathrm{AA}$ & \\
\hline Segment 2 & VP2 (T2) & $98 \mathrm{kDa}$ & Inner shell & Segment 2 & VP3 (T2) & 105 kDa & Inner shell \\
\hline $2747 \mathrm{bp}$ & YP_052943 & $890 \mathrm{AA}$ & & $2856 \mathrm{bp}$ & YP_460039 & $925 \mathrm{AA}$ & \\
\hline Segment 3 & VP3 & $74 \mathrm{kDa}$ & Outer shell & Segment 3 & VP2 & 104 kDa & Outer shell \\
\hline 2024 bp & YP_052944 & $654 \mathrm{AA}$ & & $2747 \mathrm{bp}$ & YP_460040 & $881 \mathrm{AA}$ & \\
\hline Segment 4 & VP4 (CaP) & $74 \mathrm{kDa}$ & Capping Enzyme & Segment 4 & VP4 (CaP) & $74 \mathrm{kDa}$ & Capping Enzyme \\
\hline 2017 bp & YP_052945 & $643 \mathrm{AA}$ & & 1996 bp & YP_460041 & $646 \mathrm{AA}$ & \\
\hline Segment 5 & VP5 & $57 \mathrm{kDa}$ & Outer shell & Segment 5 & NS1 (TuP) & $64 \mathrm{kDa}$ & Forms Tubules \\
\hline $1664 \mathrm{bp}$ & YP_052946 & $517 \mathrm{AA}$ & & $1784 \mathrm{bp}$ & YP_460045 & $554 \mathrm{AA}$ & \\
\hline Segment 6 & NS1 (TuP) & $58 \mathrm{kDa}$ & Forms Tubules & Segment 6 & VP5 & $59 \mathrm{kDa}$ & Outer shell \\
\hline $1657 \mathrm{bp}$ & YP_052947 & $517 \mathrm{AA}$ & & $1695 \mathrm{bp}$ & YP_460042 & $529 \mathrm{AA}$ & \\
\hline Segment 7 & NS2 (ViP) & $51 \mathrm{kDa}$ & Viral inclusion body matrix protein & Segment 7 & NS2 (ViP) & $48 \mathrm{kDa}$ & Viral inclusion body matrix protein \\
\hline $1463 \mathrm{bp}$ & YP_052948 & $462 \mathrm{AA}$ & & 1613 bp & YP_460046 & $435 \mathrm{AA}$ & \\
\hline Segment 8 & VP7 (T13) & $41 \mathrm{kDa}$ & Inner shell & Segment 8 & VP7 (T13) & $40 \mathrm{kDa}$ & Inner shell \\
\hline $1256 \mathrm{bp}$ & YP_052949 & $379 \mathrm{AA}$ & & $1180 \mathrm{bp}$ & YP_460044 & $353 \mathrm{AA}$ & \\
\hline Segment 9 & VP6 (Hel) & $26 \mathrm{kDa}$ & ssRNA and dsRNA binding helicase & Segment 9 & VP6 (Hel) & $37 \mathrm{kDa}$ & ssRNA and dsRNA binding helicase \\
\hline $764 \mathrm{bp}$ & YP_052950 & $232 \mathrm{AA}$ & & $1071 \mathrm{bp}$ & YP_460043 & $334 \mathrm{AA}$ & \\
\hline Segment 10 & NS3 & $24 \mathrm{kDa}$ & Glycoprotein & Segment 10 & NS3 & $28 \mathrm{kDa}$ & Glycoprotein \\
\hline $764 \mathrm{bp}$ & YP_052951 & $224 \mathrm{AA}$ & & 819 bp & YP_460047 & $255 \mathrm{AA}$ & \\
\hline
\end{tabular}




\section{BRDV (tick-transmitted)}

Segment 1

Segment 2

$\begin{array}{ll}\text { VP2 (T2) } & 103 \mathrm{kDa} \\ \text { P35934 } & 908 \mathrm{AA}\end{array}$

Segment 3

Segment 4

VP4 †

$63 \mathrm{kDa}+$

Segment 5

$1658 \mathrm{bp}$

VP5

P21230

$53 \mathrm{kDa}$

$480 \mathrm{AA}$

Segment 6

$1714 \mathrm{bp}$

NS1 (TuP)

2115436A

$60 \mathrm{kDa}$

Segment 7

\section{VP7}

(T13)

P35935

$40 \mathrm{kDa}$

$356 \mathrm{AA}$
Outer shell

Segment 4

LIPV (tick-transmitted)

Segment 1

$3892 \mathrm{bp}$

VP1 (Po)

ADM88603

$146 \mathrm{kDa}$

$1284 \mathrm{AA}$

Segment 2

VP2 (T2)

ADM88604

$103 \mathrm{kDa}$

$908 \mathrm{AA}$

Segment 3

\section{Outer shell}

Segment 5

Formes Tubules

Segment 6

1509 bp +十

Segment 7

Inner shell
Segment 8

Segment 9

Segment 10

Glycoprotein

P32555

$205 \mathrm{AA}$

Outer and inner shell proteins are labeled in bold. GenBank and SwissProt accession numbers are indicated.

t: [12,13], GenBank entry missing.

t†: partial sequence.

Segment 8

\section{Segment 9}

Segment 10 
might be involved in membrane fusion and penetration [14]. TRBV and KEMV also encode VP5 on segment 6 . The highest similarity of TRBV VP5 is to LIPV VP5 (95.6\%), again encoded by segment 6 [7]. However, VP5 of BRDV is described as encoded by segment 5 [15]. Since in the classification of the viral genome segments bigger segments have smaller segment numbers, and the size of BRDV segment 6 (1714 bp) encoding the NS1(TuP) [16] is larger than the size of BRDV segment 5 (1658 bp) encoding VP5, a reassignment of BRDV segment 5 and 6 (a vice versa switch) seems necessary.

To summarize, it would be much more helpful if the nomenclature of the viral proteins in orbiviruses would reflect the sequence homology and functional relationship rather than protein size or encoding segment size, since the sizes of the orbivirus genome segments sometimes only differ slightly, which leads to even closely related viruses such as TRBV and KEMV encoding VP2 and NS1 (TuP) on different genome segments. We therefore suggest the following concise nomenclature based on the type species BTV and on sequence homology and functional characteristics independent of segment or protein size: VP1(Pol), VP2, VP3(T2), VP4(CaP), VP5, VP6(Hel), VP7 (T13), NS1(TuP), NS2(ViP), NS3.

\section{Competing interests}

The authors declare that they have no competing interests.

\section{Author's contributions}

MD and MW wrote the paper. Both authors read and approved the final manuscript.

\section{Acknowledgments}

This work was supported by the Federal Ministry of Education and Research (BMBF), grant number 01KI0710, "Research on Zoonotic Infectious Diseases" program, "Emerging arthropode-borne viral infections in Germany: Pathogenesis, diagnostics and surveillance" and the BMBF funded research program "Potential release-oriented biothreat emergency diagnostics (P.R.O.B. E)" for civil security of the German Federal Government as part of the hightech strategy for Germany.

Received: 1 February 2012 Accepted: 24 July 2012

Published: 21 August 2012

\section{References}

1. Attoui H, Mertens PPC, Becnel J, Belaganahalli S, Bergoin M, Brussaard CP, Chappell JD, Ciarlet M, del Vas M, Dermody TS, et al: Orbiviruses, Reoviridae. In Virus taxonomy ninth report of the international committee on taxonomy of viruses. Edited by King AMQ, Adams MJ, Carstens EB, Lefkowitz EJ. Elsevier: Adacemic Press; 2011:592-603.

2. Attoui H, Jaafar FM, Belhouchet M, Aldrovandi N, Tao SJ, Chen BQ, Liang GD, Tesh RB, de Micco P, de Lamballerie X: Yunnan orbivirus, a new orbivirus species isolated from Culex tritaeniorhynchus mosquitoes in China. J Gen Virol 2005, 86:3409-3417.

3. Gorman BM: Variation in orbiviruses. J Gen Virol 1979, 44:1-15.

4. Moss SR, Jones LD, Nuttall PA: Comparison of the major structural core proteins of tick-borne and culicoides-borne orbiviruses. J Gen Virol 1992, 73:2585-2590

5. Doherty RL, Carley JG, Murray MD, Main AJ, Kay BH, Domrow R: Isolation of arboviruses (Kemerovo-Group, Sakhalin-Group) from ixodes-uriae collected at Macquarie Island, Southern Ocean. Am J Trop Med Hyg 1975, 24:521-526.

6. Gorman BM, Taylor J, Morton HC, Melzer AJ, Young PR: Characterization of nugget virus, a serotype of the kemerovo group of orbiviruses. Aust J Exp Biol Med Sci 1984, 62:101-115.

7. Belhouchet M, Jaafar FM, Tesh RB, Grimes J, Maan S, Mertens P, Attoui H: Complete sequence of the Great Island virus and comparison with the T2 and outer-capsid proteins of Kemerovo, Lipovnik and Tribec viruses (genus Orbivirus, family Reoviridae). J Gen Virol 2010, 91:2985-2993.

8. Major L, Linn ML, Slade RW, Schroder WA, Hyatt AD, et al: Ticks associated with Macquarie Island penguins carry arboviruses from four genera. PLOS One 2009, 4:e4375.

9. Attoui H, Stirling JM, Munderloh UG, Billoir F, Brookes SM, Burroughs JN, de Micco P, Mertens PPC, de Lamballerie X: Complete sequence characterization of the genome of the St Croix River virus, a new orbivirus isolated from cells of Ixodes scapularis. J Gen Virol 2001, 82:795-804.

10. Dilcher M, Hasib L, Lechner M, Wieseke N, Middendorf M, Marz M, Koch A, Spiegel M, Dobler G, Hufert FT, Weidmann M: Genetic characterization of Tribeč virus and Kemerovo virus, two tick-transmitted human-pathogenic Orbiviruses. Virology 2012, 423:68-76.

11. Attoui H, Mendez-Lopez MR, Rao SJ, Hurtado-Alendes A, Lizaraso-Caparo F, Jaafar FM, Samuel AR, Belhouchet M, Pritchard LI, Melville L, et al: Peruvian horse sickness virus and Yunnan orbivirus, isolated from vertebrates and mosquitoes in Peru and Australia. Virology 2009, 394:298-310.

12. Moss SR, Nuttall PA: Subcore-like and core-like particles of Broadhaven Virus (Brdv), a tick-borne orbivirus, synthesized from baculovirusexpressed Vp2 and Vp7, the major core proteins of Brdv. Virus Res 1994, 32:401-407.

13. Schoehn G, Moss SR, Nuttall PA, Hewat EA: Structure of Broadhaven virus by cryoelectron microscopy: Correlation of structural and antigenic properties of Broadhaven virus and bluetongue virus outer capsid proteins. Virology 1997, 235:191-200.

14. Zhang X, Boyce M, Bhattacharya B, Zhang XK, Schein S, Roy P, Zhou ZH: Bluetongue virus coat protein VP2 contains sialic acid-binding domains, and VP5 resembles enveloped virus fusion proteins. Proc Natl Acad Sci U S A 2010, 107:6292-6297.

15. Moss SR, Fukusho A, Nuttall PA: Rna segment 5 of Broadhaven virus, a tick-borne orbivirus, shows sequence homology with segment- 5 of bluetongue virus. Virology 1990, 179:482-484.

16. Moss SR, Nuttall PA: Comparison of the nonstructural protein, Ns1, of tickborne and insect-borne orbiviruses. Virus Res 1995, 36:287-292.

doi:10.1186/1743-422X-9-166

Cite this article as: Dilcher and Weidmann: Confusions in orbivirus protein classification. Virology Journal 2012 9:166.

\section{Submit your next manuscript to BioMed Central and take full advantage of:}

- Convenient online submission

- Thorough peer review

- No space constraints or color figure charges

- Immediate publication on acceptance

- Inclusion in PubMed, CAS, Scopus and Google Scholar

- Research which is freely available for redistribution 